\title{
Posterior reversible encephalopathy syndrome due to seronegative systemic lupus erythematosus
}

\author{
Sawan Verma, Irfan Yousuf, Mushtaq Ahmad Wani, Ravouf Asimi, Sheikh Saleem, Mudasir Mushtaq, \\ Irfan Shah, Skeikh Nawaz, Riyaz Ahmad Daga \\ Department of Neurology, Sher-e-Kashmir Institute of Medical Sciences, Soura, Srinagar 190011, Jammu and Kashmir, India.
}

\section{A B S T R A C T}

Posterior reversible encephalopathy syndrome (PRES) is a neurotoxic state coupled with a unique computed tomography or magnetic resonance imaging (MRI) appearance. Recognized in the setting of a number of complex conditions (preeclampsia/eclampsia, allogeneic bone marrow transplantation, organ transplantation, autoimmune disease and high-dose chemotherapy) in the imaging, clinical and laboratory features of this toxic state are becoming better elucidated. We are presenting a case of PRES due to seronegative systemic lupus erythematosus, with MRI findings of diffuse vasogenic edema.

Key words: Posterior reversible encephalopathy syndrome, seronegative systemic lupus erythematosus, vasogenic edema

\section{INTRODUCTION}

Posterior reversible encephalopathy syndrome (PRES) is a clinicoradiological entity that was well-described by Hinchey et al. ${ }^{[1]}$ in 1996. This condition has been designated by a variety of names (reversible posterior leukoencephalopathy syndrome, reversible posterior cerebral edema syndrome, and reversible occipital parietal encephalopathy). PRES is now the accepted term but has been challenged recently based on the risk of neurological impairment and up to $15 \%$ mortality rate. ${ }^{[2]}$ The most common clinical symptoms and signs are headache, altered alertness and behavior ranging from drowsiness to stupor, seizures, vomiting, mental abnormalities including confusion and diminished spontaneity and speech, and abnormalities of visual perception. ${ }^{[1]}$ Hypertension is the most commonly identified cause of PRES, followed by medications, eclampsia and other systemic factors. The pathophysiology of hypertension related to PRES is due to a failure of cerebrovascular autoregulation, which in turn results in vasogenic edema. Nonhypertensive PRES may be due to an autoimmune or immune response to various stimuli.

\begin{tabular}{|l|l|}
\hline \multicolumn{3}{|c|}{ Access this article online } \\
\hline Quick Response Code: & \\
\hline & Website: \\
& www.nnjournal.net \\
& \\
&
\end{tabular}

Usually, it is a reversible phenomenon, as indicated by the name, but if not recognized early and treated appropriately, permanent brain injury may ensue. ${ }^{[3]}$

\section{CASE REPORT}

A 55-year-old female, known case of hypertension, hypothyroidism, old treated pulmonary tuberculosis (12 years back) admitted with the chief complaints of generalized swelling, recurrent vomiting and weakness of all 4 limbs for 3 months and altered sensorium for 1-week duration. Patient was initially evaluated outside and following studies elicited no abnormalities: complete blood count, kidney function tests, liver function tests, blood glucose, and electrolytes. Abdominal ultrasonogram detects bilateral pleural effusion (right $>$ left side) and free fluid in pelvis. Nerve conduction studies of all 4 limbs revealed mild asymmetrical large fiber, motor axonal neuropathy in lower limbs > upper limbs, whereas electromyography revealed acute denervation in proximal and distal muscles of both lower limbs. Magnetic resonance imaging (MRI) of spinal cord showed spondylotic changes with mild disc dehydration and posterior disc bulge at L4-L5 level. Vasculitic profile, including antinuclear antibody (ANA) three was negative. She was managed with intravenous (i.v.) fluids, levothyroxine, antihypertensive, and i.v. methylprednisolone ( $1 \mathrm{~g}$ daily for 5 days). She improved clinically to some extent and was discharged. Soon within 2 days after discharge, she became confused and agitated and was brought to our department as a case of encephalopathy.

Corresponding Author: Dr. Sawan Verma, Department of Neurology, Sher-e-Kashmir Institute of Medical Sciences, Soura, Srinagar 190011, Jammu and Kashmir, India. E-mail: verma.sawan@gmail.com 
A detailed history revealed past history of arthralgias and photosensitivity, for which she was not on any treatment. On clinical examination, she was in grade 3 encephalopathy with Glasgow Coma Scale (GCS) of 8/15, hemodynamically stable, with generalized areflexia. Rest of the systemic examination was normal. Serum urea and creatinine levels were on higher side (serum urea $=88$, serum creatinine $=1.9$ ), whereas rest of the baseline investigation and biochemistry were within the normal limits. Ultrasound abdomen showed bilateral raised cortical echogenicity, with mild ascites. Repeat vasculitic profile including ANA, anti-ds-DNA, Perinuclear anti-neutrophil cytoplasmic antibodies (P-ANCA), cytoplasmic antineutrophil cytoplasmic antibodies (C-ANCA), anticentromere were negative. Noncontrast computed tomography head was suggestive of diffuse brain edema [Figure 1], while MRI brain showed diffuse bilateral edema in white matter predominantly in occipital region [Figures 2-4]. Cerebrospinal fluid was acellular with raised protein (92 mg/dL) and glucose (126 mg/ dL). She was restarted with i.v. methylprednisolone (1 g daily) for 5 days and followed by oral prednisolone ( $1 \mathrm{mg} / \mathrm{kg}$ body weight). After 2 weeks, the patient's condition had improved to the point that she was conscious with GCS of 15/15, could ambulate without assistance and showed nearly normal strength in all

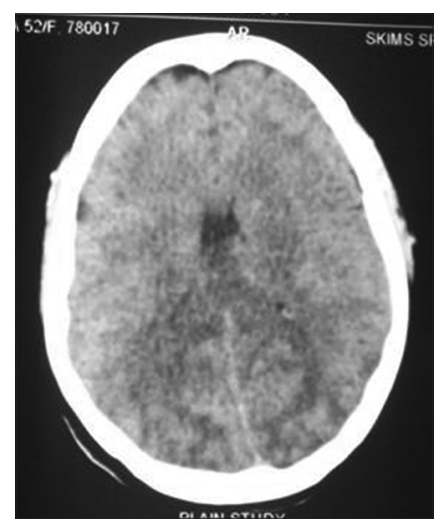

Figure 1: Noncontrast computed tomography head suggestive of diffuse brain edema

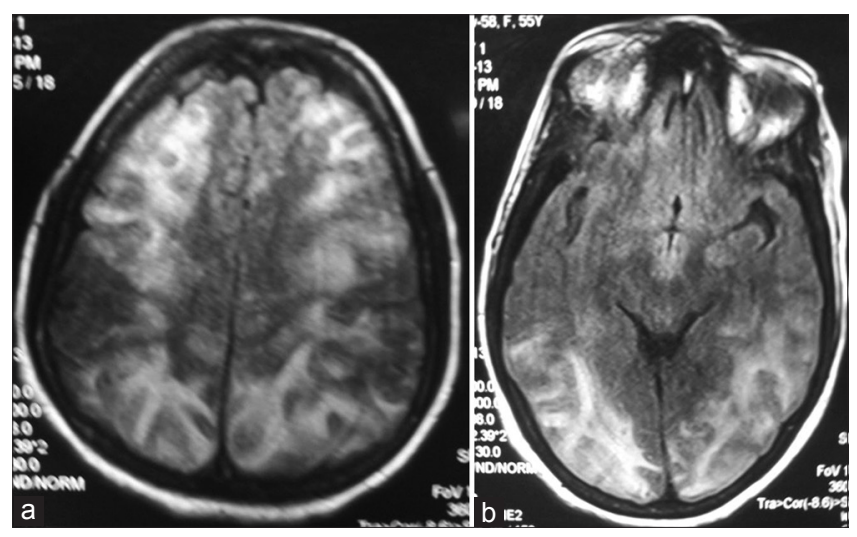

Figure 3: Fluid attenuation inversion recovery image showing (a) suggestive of diffuse white matter edema and (b) edema in posterior circulation region
4 limbs. Follow-up MRI [Figure 5] showed significant resolution of white matter edema. Hence, on the basis of arthralgias and photosensitivity in past and features of polyserositis, renal impairment and neurological dysfunction in the form of encephalopathy, a likely diagnosis of seronegative systemic lupus erythematosus (SLE) presenting first time as PRES, was established. Patient is presently on oral tapering dose of steroids along with supportive treatment.

\section{DISCUSSION}

The exact pathophysiological mechanism of PRES remains uncertain. To date, three hypotheses have been proposed, which include: (1) cerebral vasoconstriction with subsequent infarcts of the brain, (2) failure of cerebral autoregulation with consequent vasogenic edema, and (3) endothelial damage with disruption of the blood-brain barrier (BBB) causing fluid and protein transudation in the brain. The pathophysiology of PRES in SLE is also less well understood. In most cases of SLE-related PRES, immunosuppresants used to treat the SLE were suggested as causative factors, though lupus itself or SLE-related hypertension, antiphospholipid antibodies or renal failure might also be contributive. Abnormal endothelial activation, dysfunction and leukocyte tracking have recently been documented to

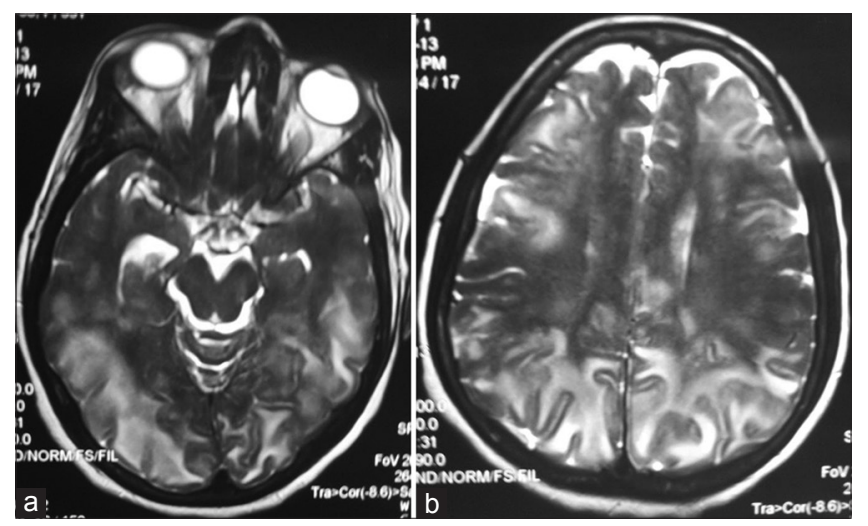

Figure 2: T2-weighted magnetic resonance imaging showing. (a) edema in posterior circulation region and; (b) diffuse white mater edema

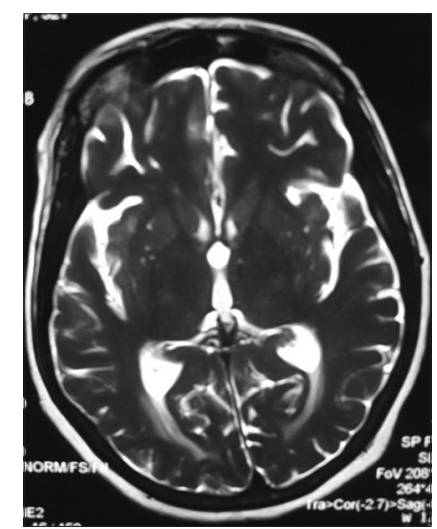

Figure 4: Repeat T2-weighted magnetic resonance imaging showing significant reduction in brain edema 


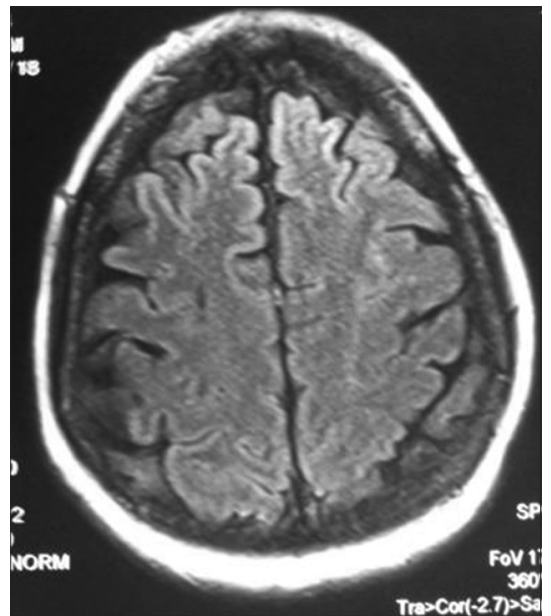

Figure 5: Repeat fluid attenuation inversion recovery image showing resolution of white matter edema in bilateral cerebral hemisphere

cause brain and systemic hypoperfusion, which may be causative factors for PRES in SLE. On the other hand, endothelial cell activation is one of the pathogenic hallmarks of neuropsychiatric SLE (NPSLE). It usually occurs after exposure to interleukin 1 (IL-1) and tissue necrotic factor- $\alpha$ (TNF- $\alpha$ ), and may be enhanced by local release of IL-1 and IL-6. SLE patients with high SLE disease activity index have increased serum levels of TNF- $\alpha$ and other pro-inflammatory cytokines that may stimulate endothelial cells of intracranial vessels and astrocytes to produce nitric oxide, causing $\mathrm{BBB}$ damage and plasma leakage. In some cases the endothelial dysfunction together with hemodynamic factors may allow the leakage of blood plasma and large amounts of red blood cells resulting in secondary parenchymal hematoma. Histopathology showed the PRES manifestation result from NPSLE were due to focal cerebral edema associated with blood vessel injury and ischemic changes, although in many cases histopathology did not demonstrate specific lesions. SLE patients might develop reversible focal neurological deficits, which responded to steroid therapy. ${ }^{[4]}$

Even though the classical neurolupus includes seizures and psychosis, a number of other features such as myelopathy, optic neuropathy, meningitis, cognitive dysfunction, and cerebral infarction could be seen in SLE. PRES has been claimed as a particular form of neurological manifestation of SLE with characteristic MRI findings and a usual good outcome. Antihypertensive, antiepileptic, and supportive care are the mainstay of treatment. ${ }^{[5]}$

In some cases, the diagnosis of PRES remains in doubt. In this situation, regression of the clinical and radiological abnormalities with appropriate treatment supports the diagnosis. Thus, repeated brain imaging is beneficial of diagnosis. ${ }^{[6]}$ Radiographically, PRES is heralded by relatively symmetric, reversible T2 hyperintensities affecting the posterior aspects of the brain, namely the occipital and parietal lobes. It is now known that this description is more of a general rule, and those asymmetric images can be seen, and can involve the deep grey matter as well as the frontal and temporal lobes. The advent of diffusion weighted imaging helped clarify that the MRI changes were not due to ischemia or cytotoxic edema, but due to vasogenic edema. ${ }^{[7]}$

In our case, as per diagnostic criteria for SLE, ${ }^{[8]}$ more than four well documented features were present, that is, history of arthralgias, photosensitivity, polyserositis, renal impairment and nervous system involvement. Although her vasculitic profile was negative, but her brain imaging was suggestive of diffuse white matter edema, she was treated as seronegative SLE presenting as PRES. She received pulse therapy of i.v. methylprednisolone followed by oral steroids as per body weight. Patient improves clinically and her repeat imaging, done after 6 weeks, was almost normal.

\section{REFERENCES}

1. Hinchey J, Chaves C, Appignani B, Breen J, Pao L, Wang A, Pessin MS, Lamy C, Mas JL, Caplan LR. A reversible posterior leukoencephalopathy syndrome. NEngl J Med 1996;334:494-500.

2. Legriel S, Pico F, Azoulay E. Understanding posterior reversible encephalopathy syndrome. In: Vincent JL, editor. Annual Update in Intensive Care and Emergency Medicine 2011. Berlin, Germany: Springer; 2011. p. 631-53.

3. Hedna VS, Stead LG, Bidari S, Patel A, Gottipati A, Favilla CG Salardini A, Khaku A, Mora D, Pandey A, Patel H, Waters MF. Posterior reversible encephalopathy syndrome (PRES) and CT perfusion changes. Int J Emerg Med 2012;5:12.

4. Liu B, Zhang X, Zhang FC, Yao Y, Zhou RZ, Xin MM, Wang LQ. Posterior reversible encephalopathy syndrome could be an underestimated variant of "reversible neurological deficits" in systemic lupus erythematosus. BMC Neurol 2012;12:152.

5. Marrone L, Streck Ade S, Staub HL, de Freitas CZ, Costa J, Gadonski G, Luiz Staub H. Posterior reversible encephalopathy syndrome (PRES) and systemic lupus erythematosus: report of two cases. Rev Bras Reumatol 2012;52:804-10.

6. Kadikoy H, Haque W, Hoang V, Maliakkal J, Nisbet J, Abdellatif A. Posterior reversible encephalopathy syndrome in a patient with lupus nephritis. Saudi J Kidney Dis Transpl 2012;23:572-6.

7. Graham BR, Pylypchuk GB. Posterior reversible encephalopathy syndrome in an adult patient undergoing peritoneal dialysis: a case report and literature review. BMC Nephrol 2014;15:10.

8. Tan EM, Cohen AS, Fries JF, Masi AT, McShane DJ, Rothfield NF, Schaller JG, Talal N, Winchester RJ. The 1982 revised criteria for the classification of systemic lupus erythematosus. Arthritis Rheum 1982;25:1271-7. Updated by: Hochberg MC. Updating the American College of Rheumatology revised criteria for the classification of systemic lupus erythematosus. Arthritis Rheum 1997;40:1725.

Cite this article as: Verma S, Yousuf I, Wani MA, Asimi R, Saleem S, Mushtaq M, Shah I, Nawaz S, Daga RA. Posterior reversible encephalopathy syndrome due to seronegative systemic lupus erythematosus. Neuroimmunol Neuroinflammation 2014;1(2):89-91.

Source of Support: Nil. Conflict of Interest: No.

Received: 09-02-2014; Accepted: 18-07-2014 